\title{
Testing Complex Emotion Recognition in Adults: A Gendered Comparison
}

\author{
Nahla Aljojo \\ King Abdulaziz University \\ Faculty of Computing and Information Technology, \\ Information System Department \\ P.O. Box , 132139, Jeddah, 21382, KSA
}

\author{
Huda Saifuddin \\ King Abdulaziz University \\ Arts and Humanities College, Cognitive \\ Psychology Department \\ P.O. Box 7571, Makkah, KSA
}

\begin{abstract}
Determining the integrity of emotion recognition in adults is important to our understanding of facial expressions and the learning of social skills. This study implements a new set of tasks, testing 109 individuals in Saudi Arabia with the use of the Cambridge Mindreading (CAM) Test. The test was applied to adult males and females. Results showed how people performed with regard to emotion recognition displayed by other faces. The implications of the test results will help people know their level of understanding regarding the facial expressions of others before ascertaining the appropriate suggestions, and to make the test suit our society. Additionally, this study focuses on the extent to which a person can understand the facial expressions of the people around them, and also this test can be used to help to diagnose if people suffer from Asperger autism. The authors provide a review of previous studies and a description of the approach that the authors follow, as well as concluding observations. This paper compares male and females reading facial expressions in Saudi Arabia, the result shows a significant difference between male and female participants.
\end{abstract}

\section{General Terms}

Emotion recognition, Complex emotions

\section{Keywords}

Emotion recognition; Complex emotions; Face perception; Voice perception; Theory of mind

\section{INTRODUCTION}

Facial expressions are one or more movements or positions of the muscles underneath the skin of the face. These developments pass on the passionate condition of a single person to onlookers. Outward appearances are a manifestation of nonverbal correspondence, they are an essential method for conveying social data between people, yet they also occur in many other warm-blooded animals as well as some other animal species. People can receive a facial expression intentionally or automatically, and the neural instruments in charge of controlling the outflow can vary in each case. Intentional outward appearances are regularly socially molded and follow a cortical course in the mind. Then again, automatic outward appearances are accepted as characteristic and are taken after a sub-cortical course in the cerebrum [1].

Facial recognition is regularly an enthusiastic experience for the mind and the amygdala is exceptionally included throughout the recognition process. The eyes are frequently seen as vital gimmicks of outward appearances. Perspectives - for example, squinting rate — can be utilized to show whether an individual is apprehensive or whether he or she is lying. Additionally, eye contact is perceived as an imperative part of interpersonal correspondence. Conversely, there exist societal contrasts with regard to the social respectability of maintaining eye contact or not. Beyond the extra nature of outward appearances in spoken correspondence between individuals, these assume a critical part in correspondence with gesture-based communication. Numerous expressions in gesture-based communication incorporate outward appearances in the showcase. [1]

Facial expressions are a vital part of communication. What remains funny is that most people talk about facial expressions without really know what they are. According to experts such as Baron Cohen and Paul Ekman [2], among others, these nonverbal signals make up a huge part of daily communication. From our facial expressions to our body movements, the things we do not say can nevertheless convey volumes of information. According to various researchers, such as Lanzetta [3], among others, body language is thought to account for between 50-70 percent of all communication. Understanding body language is important, but it is also essential to note other cues such as context and to look at signals as a group rather than focusing on a single action. Inde3ed, we should endeavor to learn more about some of the things to look for when you are trying to interpret body language [4].

The significance of understanding a facial expression follows from the fact that communicative interaction assimilates both verbalized and non-verbalized means of conveying information. When communicating with others we often use facial expressions. It remains significant to comprehend such delicate signs as a greater component of the communicative phenomenon. An elementary expression of a smile may suggest that it is concurrence with a piece of conveyed information, or in agreement with the stating of the information itself. Furthermore, a frown can suggest that one is experiencing contentiousness or belligerence toward a concept. Understanding facial expressions and their meaning is an important part of the communication process [5].

Generally there can be said to be no ubiquity of consensus or understanding of facial expression that affect societal interactions, and this remains an aim for those trying to formulate and enact such a phenomenon. One needs to assess the facial action regardless of whether it is happy or angry or so on; inappropriate types of behavior or feelings under normal circumstances; a general pervasive mood of unhappiness or depression; difficultly making friends ignored or rejected; incapacity to be able to form or perpetuate a relationship through anxiousness can result in damage to the person experiencing the sentiment as well as other around them, according to Levenson [6]. 
Some academics (such as Ekman [1] and Joseph \& Hager [7]) consider the internalized and externalized message of a facial expressions; facial expression suggests that the phenomenon is a considerable and multifarious group. The connection of more instinctual expressions to a sentiment is exact and is purified through alternative expressions that relate to alternative, separate sentiment. Minute and idiosyncratic alterations within a solitary expression can relate to differentiation in the experience of the subsequent sentiment. The symmetrical nature of an expression can divulge if it is spontaneous as well as emotional, according to Joseph \& Hager [7].

A number of scientific professionals expound upon the many hypotheses that are assimilated into the educational rubric of emotional recognition within social psychology as well as human neuropsychology. Indeed, Darwin [8] devised a triumvirate of principles that were utilized in the assessment of expressions and argued for significant attention to be given to the assessment of the active component of various muscles to determine a given expression. The ubiquitous grouping of muscles found in the face as well as exacting emotive expression (such as happiness, sadness, anger, fear, surprise, disgust, interest). In accordance to some academics the expression of one's face may indeed be brought about as a result of being galvanized by emotive incentive, and thus can have three alternative eventualities with regard to societal interactivity. Some concur that human facial muscles and facial expression have developed into communicative informational entities into receiving ones; thereby altering their behavioral characteristics, as emotive expressions in people may also be used to mislead. An additional theoretical role of the emotive expression is to facilitate the sender of the expression with data regarding their own current disposition [9].

The Emergent Emotion Model is a biologically inspired model allowing creation of a believable guide. It regulates the guide's internal states and these subsequently act the guide's behavior and processing strategy. The emotions of the Active Guide are not explicitly denned but emerge from modulation of information processing. In the current version of the Active Guide, animation of the facial expressions is performed by swapping facial feature frames. Even with such a limited number of frames per feature, vast combinations of facial expressions were generated, implying that an inmate variation could be achieved if morphing is possible [10].

Bowers [11] discovered that patients that had injury to the right hemisphere of their face found it more challenging to expound upon mental pictures of facial sentiments or expressions, though they experienced no issue with the generation of pictures displaying a neutral sentiment.

Others, such as Ley and Bryden [12], presented cartoons of five alternative persons, each of which showed a different expression; these altered from bad or negatively-inclined expressions to good or positively-inclined expressions. With the use of a tachistoscope, the researchers presented the cartoons for a small period of time, discovering that on presentation of a formidable or extreme emotional expression, the patient's right hemispheres interpreted them with greater exactness [12].

Still others, such as Eibl-Eibesfeldt [13], have suggested that people's musculature and related expressions have progressed through evolutionary means to convey information to peers in order to try and alter behavior. The means to convey and comprehend a set of basis emotive expressions appears to be ubiquitous.

Mackey [14] stipulated that if people's facial expressions, with regard to their sentiment, progressed within evolution for the intention of conveying information and communicative ends then it would be assumed that, as a result of such causes, one would expect the sender's emotional state to be of the greatest informational importance.

The most sound evidence with regard to the facial feedback hypothesis unto the present time originated from Lanzetta [3]. who discovered that persons who tried to conceal the agony of a shock that they were experiencing displayed a lower skin conductivity as well as their subjectivist gauging of pain; furthermore, that those who tried to convey the experience of agony displayed increases with regard to each of these measures. (Baron-Cohen 1 [15]) compared male and female in reading facial expressions, the result shows Females were significantly better than males at recognizing emotions from faces, regardless of diagnosis.

Extant research assessing the emotional alterations of females and males is abundant. The aim of this study is to assess the emotive expressivity of both males and females within the remit of social networking; through websites like Facebook and Twitter. Information was assimilated with the assessment of posts, 'tweets', as well as status updates and comments. The information was extrapolated from a sample population of Australian males and females with the results of the findings of the information being expounded upon and deliberated over. A statistically important grouping of research information has discovered that females are the more emotively-expressive sex within the remit of facial communication, according to Parkins (2012).

This paper has been designed with some special techniques, such as facial action coding system (FACS), with the primary goal being comprehensiveness. The Cambridge Mindreading (CAM) Test was employed as a technique that can measure all possible, visible facial actions in order to help diagnose and determine whether a person has some difficultly in understanding people's expressions. also This paper also compares males and females in their reading of facial expressions of other people around within Saudi Arabia.

The authors choose a different sample of males and females from Nafisa Shams Academy, King Abdul Aziz University and some randomly selected families to measure people's ability to understand different facial expressions of others. Then the authors subjected them to a test including 50 questions especially aimed at acknowledging facial expressions before the authors analyzed the results using an average line, which will be expounded upon below. The most optimal solution is used featured an exam System used by normal people to help them know different facial expression. The advantages of this solution are time and money-saving, providing expert opinions, which can be used anytime anywhere. 


\section{THE CAMBRIDGE MINDREADING (CAM) FACE-VOICE BATTERY}

This section outlines the Cambridge Mindreading (CAM) Face-Voice. The CAM battery assesses a grouping of 20 emotional concepts, extrapolated from the taxonomic (stipulated above), which is representing 18 form the 24 emotive groupings, thereby addressing a wide selection of emotions and states of mind, though retaining short for administrative expediency. Inclusion of all 412 emotive statuses would have resulted in an impossibly difficult and untimely test for a solitary sitting Battery (adapted from Baron-Cohen [15]).

The battery includes two tasks: emotion recognition in the face and emotion recognition in the voice. Each of these tasks has fifty questions, in which the participant is either watching 3-5 second silent clips of actors portraying an emotion (facial task), or listening to short sentences, spoken in a particular emotional intonation (vocal task). Subsequent to seeing or hearing the recording of the voice, the sample individual is given four different adjectives and then required to "select the most accurate descriptive word that matches the emotion conveyed by that person". To ensure that the selected ideas are acquired from the adult emotive catalogue, they were chosen from the higher echelons of the taxonomic grouping; six concepts from level four (concepts understood by typical 15-16 year- olds), 13 concepts from level 5 (understood by typical 17-18 year-olds) and one concept from level six (words understood by less than 75\% of typical 17-18 yearolds). A more exacting explanation of the process strata validity procedure can be obtained from alternative sources (Baron-Cohen et al., submitted). The intention of the examination is to try and assess whether or not those who are deemed to be autistic experience challenges in reading or understanding other's emotive expressions, particularly complex expressions. Due to the fact that this component of their communicative and social skill-set and comprehending such multifaceted emotional states, it is inclusive of positive and negative emotions as well as subtle and intense emotional states. However, the purpose of this research is to examine the 'ordinary' individual by focusing upon facial emotion recognition.

Four alterative stages may be extrapolated from the CAM:

1. The overall extent of the recognition of an emotion: The sum of all the questions answered correctly; the score is between 0 and 100, explaining the general feelings and recognizing a mental state. Any score greater than 35 presents an opportunity in the above-mentioned $\mathrm{p}<.01$ level (binomial test).

2.Facial emotion recognition result: The sum of all items that were correctly answered in the face of items; the score is between 0 and 50. Any score greater than 20 presents an opportunity in the above-mentioned $\mathrm{p}<.01$ level (binomial test).

3. Voice recognition degrees of passion: The sum of all items answered correctly in the voice of items; the score is between 0 and 50. Any score greater than 20 presents an opportunity in the above-mentioned $\mathrm{p}<.01$ level (binomial test).
4. Recognized concepts correctly: Concepts can be studied in two ways; total of concepts recognized correctly; the score is between 0 and 20 (any score greater than 2 presents an opportunity in the above-mentioned $\mathrm{p}<.01$ level, according to the binomial test); or concepts certain answered correctly, analyzed individually / by subgroups (e.g. positively or negatively) (Simon Baron-Cohen [17]).

\section{METHOD}

\subsection{Participants}

The total number of participants was one-hundred and nine normal adults (26 males and 83 females) aged 14-60 yearsold. Participants in this research were mostly taken from King Abdul-Aziz University, Nafisa Shams Academy. All participants are normal adults, whether they are students or in full- or part-time employment.

\subsection{Instruments}

The task face recognition (which was taken from Cambridge Mindreading (CAM) Face-Voice Battery) was created and run on a personal computer, using compatible software. Before the test, the authors started with instructional information concerning the aims of the test and the time required to finish it. As part of the examination, the videos were shown in a stationary array. The process involved non-audio video segments of acting adults of both genders and of alternative demographical origin, displaying the emotive facial expressions (Note: shoulders were also visible). It can be seen below (see Table 1) that this included a wide array of emotive states and states of mind though the process nevertheless was kept temporally restricted for administrative purposes.

Table 1: The Cambridge Mindreading (CAM) Face-Voice Battery (adapted from Baron-Cohen et al., 2004).

\begin{tabular}{|l|l|l|}
\hline Emotion & Resentful & Repeated 3 times \\
\cline { 2 - 3 } & Stern & Repeated 3 times \\
\cline { 2 - 3 } & Grave & Repeated 2 times \\
\cline { 2 - 3 } & Subdued & Repeated 2 times \\
\cline { 2 - 3 } & Exonerated & Repeated 3 times \\
\cline { 2 - 3 } & Uneasy & Repeated 2 times \\
\cline { 2 - 3 } & Empathic & Repeated 3 times \\
\cline { 2 - 3 } & Vibrant & Repeated 2 times \\
\cline { 2 - 3 } & Lured & Repeated 3 times \\
\hline Subservient & Repeated 2 times \\
\cline { 2 - 3 } & Appalled & Repeated 3 times \\
\cline { 2 - 3 } & Confronted & Repeated 3 times \\
\cline { 2 - 3 } & Intimate & Repeated 2 times \\
\cline { 2 - 3 } & Insincere & Repeated 2 times \\
\cline { 2 - 3 } & Appealing & Repeated 2 times \\
\cline { 2 - 3 } & Mortified & Repeated 2 times \\
\cline { 2 - 3 } & Guarded & Repeated 2 times \\
\cline { 2 - 3 } & Distaste & Repeated3 times \\
\cline { 2 - 3 } & Nostalgic & Repeated 3 times \\
\cline { 2 - 3 } & Reassured & Repeated 3 times \\
\hline
\end{tabular}

The (CAM) test consisted of watching 50 videos, each video about 3-7 seconds of actors silently showing various facial expressions. The patients were given four options for each video that described the actor's expressions.

When a user enters The (CAM) Test, one video will come out on the screen with four choices, and the user must choose one choice so they are able to move to the next question. After 
finishing all 50 questions, and according to user answers, the system will then calculate how many right and wrong answers the subject has given, before telling the user if they experience difficulty understanding facial expressions. The system calculated the corrected answers questions for each participant.

The research authors have devised an online website that assimilated the test procedure and findings. The examination was shown to the members of the sample group with the use of a computer screen following access had been granted to the web domain, with the employment of a web browser. An instructional page was displayed to the participants and a series of practice clips were shown prior to the examination itself. A brief defining explanation was presented to the sample group members before the examination to instruct them of the examination and its intention. Assistance was given to the participants at all times and in all ways possible throughout the examination, within which there were no temporal constraints. The clips were then shown to the participants at random.

Participants were encouraged to ensure that they understand all the words describing each emotion, and help was given to them in case of uncertainty. In addition, the response time was unrestricted and unmeasured.
If an individual correctly answered fewer questions than average then she or he is deemed to have difficulty recognizing and understanding complex expressions. As this effects their communications with others and truer social skills, it is important to understand the extent of these difficulties, and the results may be useful in the evaluation of the problem to ensure they obtain suitable healthcare.

\subsection{Procedure}

In the case of every video, four potential responses were presented on the screen, after which verbalized expressions were shown.

Participants were then asked to choose a number from 1-4 and select the answer which describes the actor's facial expression most accurately. Then the authors played the next video in 30 seconds after the participant select their answer to allow participants to prepare for the next video. Test scores ranged from 0-50. In addition, several other measures were taken. The section below expounds upon the means by which the Face task was conducted:

\section{- Sing up:}

In this page, the user was required to fill in the registration form and create an account before he/she start the test (see Figure 1)
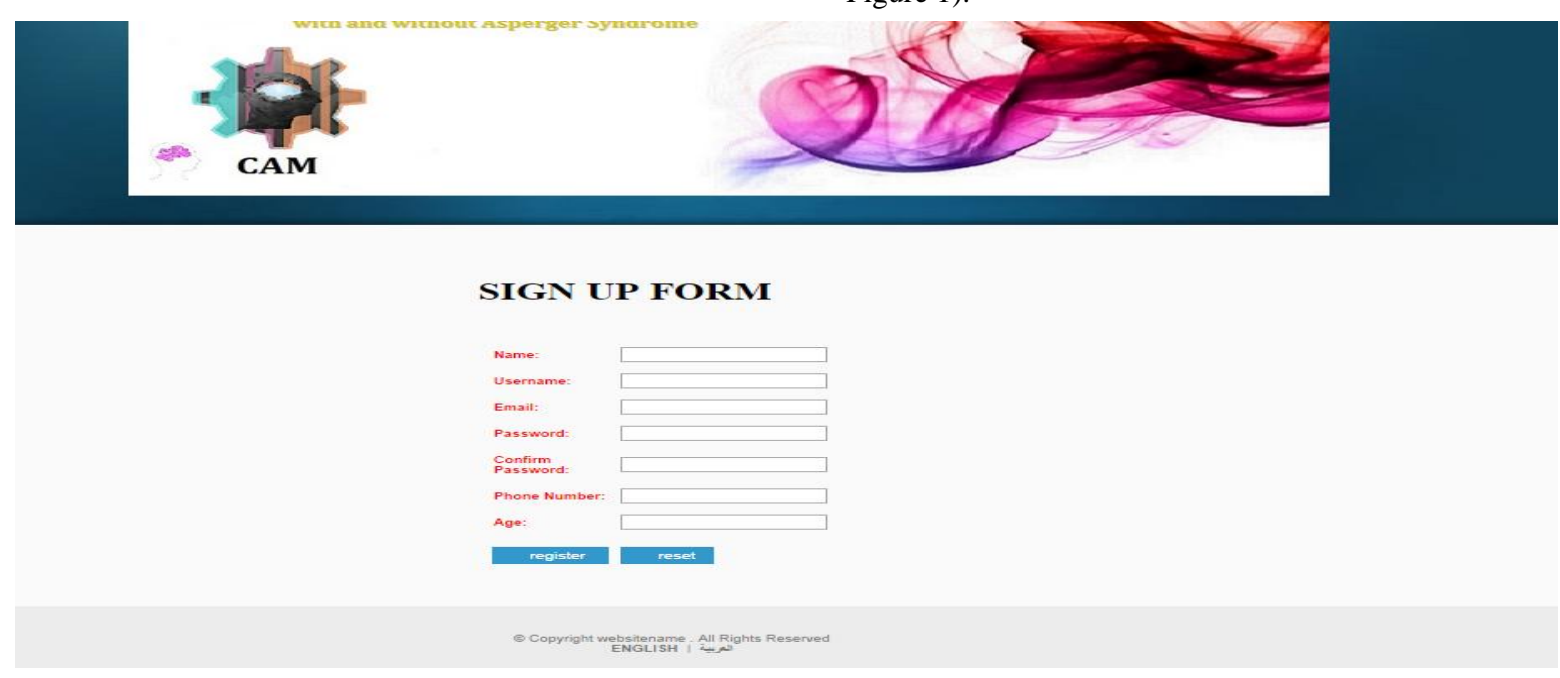

Figure 1. (CAM) sing up

- $\quad$ Start the test: The "start test" page was as shown in Figure 2, and this page contains asigninformandthebutton

that directs the user to the sign in form as well as a brief description of the test.

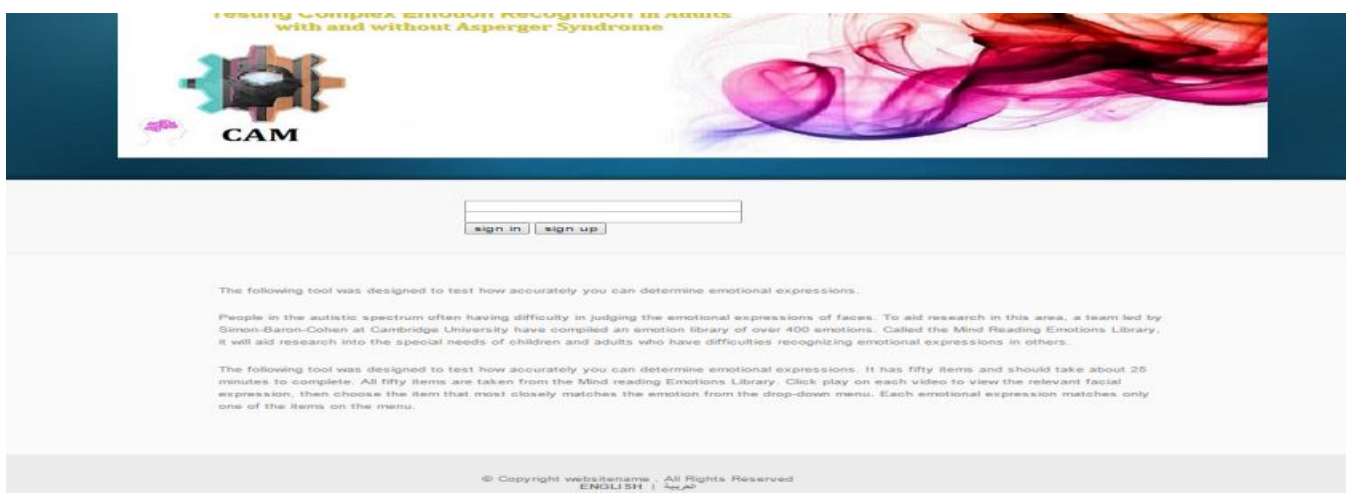

Figure 2. Start test page 
- $\quad$ Test page:

The test page contains the test, and displays one video by time with four choices; it displays the name of the current user (see Figure 3). After answering all the 50 questions, the system then calculated the correct answers and displayed the all information of user (see Figure 4).

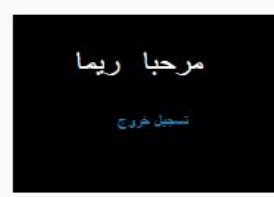

Figure 3. Test page

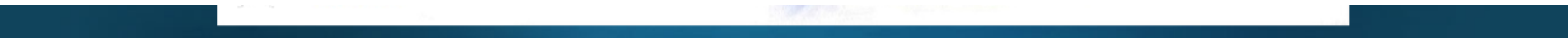

\section{USER INFORMATION}

\begin{tabular}{|l||c|}
\hline User_Id: & 1340372 \\
\hline User_Name: & reema \\
\hline Email: & reema.asaad@yahoo,com \\
\hline Phone_Number: & $05686 * * * * * *$ \\
\hline Age: & 22 \\
\hline Gender: & female \\
\hline Number of correct answers: & 37 \\
\hline
\end{tabular}

Figure 4. User information in detailed page

\section{RESULTS}

Based on the analysis of the answers the authors found that all participants (male and female groups) scored above chance ( $p<.01$, binomial test) on the facial scale. Excepting four participants who had the lowest score (the number of correct answers being fewer than 20). In addition, the mean of correct answers for both groups (male and female) was 26.64 (SD = 4.41) (see Table 2).
Table 2. Descriptive statistics of all participants

\begin{tabular}{|c|c|c|c|c|c|}
\hline $\begin{array}{c}\text { Correct } \\
\text { answer }\end{array}$ & $\mathrm{N}$ & Minimum & Maximum & Mean & $\begin{array}{c}\text { Std. } \\
\text { Deviation }\end{array}$ \\
\cline { 2 - 6 } & 109 & 15 & 41 & 26.64 & 4.41 \\
\hline
\end{tabular}

A calculation of facial scores was undertaken by counting the number of correct answers in each of the scales, as well as across the whole battery. The female eighty three adults (Mean of correct answers $=27.14, \mathrm{SD}=4.35$ ). All participants scored of female above chance $(p<.01$, binomial test) on the facial scale; excepting two female participants who had the lowest score (15 correct answers out of 50).

The 26 male adults (mean of correct answers $=25.03, \mathrm{SD}=$ 4.28). All participants scored, within the male group, above chance ( $\mathrm{p}<.01$, Binomial test) on the facial scale; excepting 
two male participants who had the lowest score (the number of correct answers being fewer than 20). The mean of the correct answers for the female group and the mean of correct answers for the male group are listed in Table 3, and it appears that the mean for the female group is higher than the mean of male group $(27.14>25.03)$.

Table 3. Descriptive Statistics of male and female groups

\begin{tabular}{|c|c|c|c|}
\hline $\begin{array}{c}\text { Correct } \\
\text { answers of } \\
\text { Male }\end{array}$ & $\mathrm{N}$ & Mean & Std. Deviation \\
\cline { 2 - 4 } & 26 & 25.04 & 4.28 \\
\hline $\begin{array}{c}\text { Correct } \\
\text { answers of } \\
\text { Female }\end{array}$ & 83 & 27.14 & 4.35 \\
\hline
\end{tabular}

Based on the independent sample, test analyses were performed on the facial scale score. Table $4 \mathrm{P}=0.033<0.05$ indicates that females were significantly better than males at recognizing emotions from faces in both groups, regardless of their diagnosis.

Table 4. Independent samples test

\begin{tabular}{|c|c|c|c|c|c|}
\hline & F & Sig & t & df & $\begin{array}{c}\text { Sig( 2 } \\
\text { tailed) }\end{array}$ \\
\hline Correct_answer & .011 & .918 & -2.183 & 107 & .033 \\
\hline $\begin{array}{c}\text { Equal variance assumed } \\
\text { Equal variance not }\end{array}$ & & & -2.179 & 42 & .035 \\
\hline
\end{tabular}

\section{DISCUSSION}

This study handles many of important issues regarding different facial expressions between women and men. It can help us to understand whether a person has difficulty in understand the facial expression of others, something that will impact upon his or her relationships. The authors implemented a test for normal adult individuals to measure the ability of their understanding regarding facial expressions of people around them; it is easy to use and can be used anywhere and anytime.

In discussing the results cited in the tables 2,3and 4 the following themes were evident

1.All participants (male and females) scored above chance ( $p<.01$, binomial test) on the facial scale. Excepting four participants who had the lowest score (the number of correct answers being fewer than 20).

2.All female participants scored above chance $(p<.01$, binomial test) on the facial scale; excepting two female participants with the lowest score (15 correct answers out of 50).

3.All male group participants scored above chance $(p<.01$, binomial test) on the facial scale; exiting two male participants who had the lowest score (the number of correct answers being fewer than 20).
4.Females were significantly better than males at recognizing emotions from faces, regardless of diagnosis.

\section{CONCLUSION}

This paper discussed The Cambridge Mindreading face expressions that arose from observations indicating that some people have difficulty in recognizing and understanding complex expressions. As this effects their communications and social skills, it remains important to understand the extent of these difficulties, and the results may be useful in evaluating their problem as well as help them to gain suitable healthcare. This paper compares the facial expressions of females with the facial expressions of males in Saudi Arabia. Female participants were found to acknowledge faces in a superior manner to males, irrelevant of the prior diagnosis.

\section{ACKNOWLEDGMENTS}

We would like to thank Reema Asa'ad and Sondos Jad, who are students of the Faculty of Computing and Information Technology, computer science Department at King Abdul Aziz University., for supporting and helping in the research. We are also grateful to the Deanship of Scientific Research (DSR), King Abdulaziz University, Jeddah, for supporting and helping Academic staff in the research.

\section{REFERENCES}

[1] Ekman, P., 1999. Facial Expressions. In: Handbook of Cognition and Emotion. s.l.:s.n.

[2] Paul Ekman, Dacher Keltner, 2008. Are Facial Expressions Universal. greater good, 12 March, p. 2.

[3] Lanzetta, 1979. Effects of nonverbal dissimulation on emotional experience and autonomic arousal. Personality and social psychology, p. 11.

[4] Hill, M., 2011. Nonverbal Communication. In: Fundamentals of Communication Studies. s.l.:s.n., p. 430. [5] Glass, L., 2004. I know What You're Thinking. second ed. s.l.:jarir library.

[5] Levinson, M., 2004. Facial Expressions Test. CIO, 1 December, p. 3.

[6] Joseph C. Hager, P. E., 1983 . humen face. [Online] Available at: http://face-and emotion.com/dataface/misctext/inner_outer.html [Accessed 193 2014].

[7] Darwin, C. (1872 orig.). The expression of the emotions in man and animals. (3rd Ed.) New York: Oxford University Press. 1998.

[8] Scientists, A. g. o., 2002. Neuropsychosocial Factors in Emotion Recognition: Facial Expressions. p. 6.

[9] Mei Yii Lim, R. A., 2008. A New Approach to Emotion Generation and Expression. School of Mathematical and Computer Sciences,Heriot Watt University., p. 8.

[10] Bowers , Blonder, Feinberg and Heilman, 1991. Differential impact of right and left hemisphere lesions on facial emotion and object imagery. Brain, p. 16.

[11] Ley, R, \& Bryden, M, 1979. Hemispheric differences in recognizing faces nd emotions. Brain and language, p. 9. [13] Eibl-Eibesfeldt, 1973. The expressive behavior of the deaf-and-blind born. social communication and movement 
[12] Mackay, 1972. Formal analysis of communication processes. Non-verbal communication.

[13] Baron-Cohen, S., Golan, O., Wheelwright, S., \& Hill, J. J. (2004). Mindreading: The interactive guide to emotions. London: Jessica Kingsley Limited (www.jkp.com).

[14] Parkins, R., 2012. Gender and Emotional Expressiveness: An Analysis of Prosodic Features in
Emotional Expression. Pragmatics and Intercultural Communication.

[15] Simon Baron-Cohen, a. J. H., 2006. The Cambridge Mindreading (CAM) Face-Voice Battery:Testing Complex Emotion Recognition in Adults with and without Asperger Syndrome. Journal of Autism and Developmental Disorders, 14 February, p. 15. 